\title{
Trauben-Eiche, Baum des Jahres 2014
}

\author{
Veit Martin Dörken
}

\begin{abstract}
Cornish oak or sessile oak (Quercus petraea) is tree of the year 2014. Its biology, ecology and uses are outlined. Differences between this species and the closely related pedunculate oak (Quercus robur) are described.
\end{abstract}

\section{Zusammenfassung}

Die Trauben-Eiche (Quercus petraea) ist Baum des Jahres 2014. Ihre Biologie, Ökologie und Verwendung werden beschrieben. Abgrenzungsmerkmale zur eng verwandten Stiel-Eiche werden genannt.

\section{Einleitung}

Die Trauben-Eiche (Quercus petraea) wurde vom Kuratorium Baum des Jahres zum 26. Baum des Jahres gekürt, weil sie eine besonders langlebige und robuste Art ist. Sie ist der Stiel-Eiche (Quercus robur), die im Jahr 1989 der erste proklamierte Baum des Jahres war, sehr ähnlich, wodurch die Unterscheidung beider Arten nicht immer einfach ist. Daher führen einige Autoren (z. B. Roloff \& Bärtels 1996) die
Trauben-Eiche nur als Unterart der Stiel-Eiche (Quercus robur subsp. petraea). Mit über 1000 Jahren Lebenserwartung gehört die TraubenEiche zu den langlebigsten einheimischen Laubbaumarten. Sie wird vom Menschen vielseitig genutzt und ist auch ökologisch von hoher Bedeutung. Die Trauben-Eiche ist Lebensraum für Hunderte verschiedener Insektenarten und deren Larven. Die Eicheln werden nicht nur von Säugetieren wie Wildschweinen und $\mathrm{Na}$ -

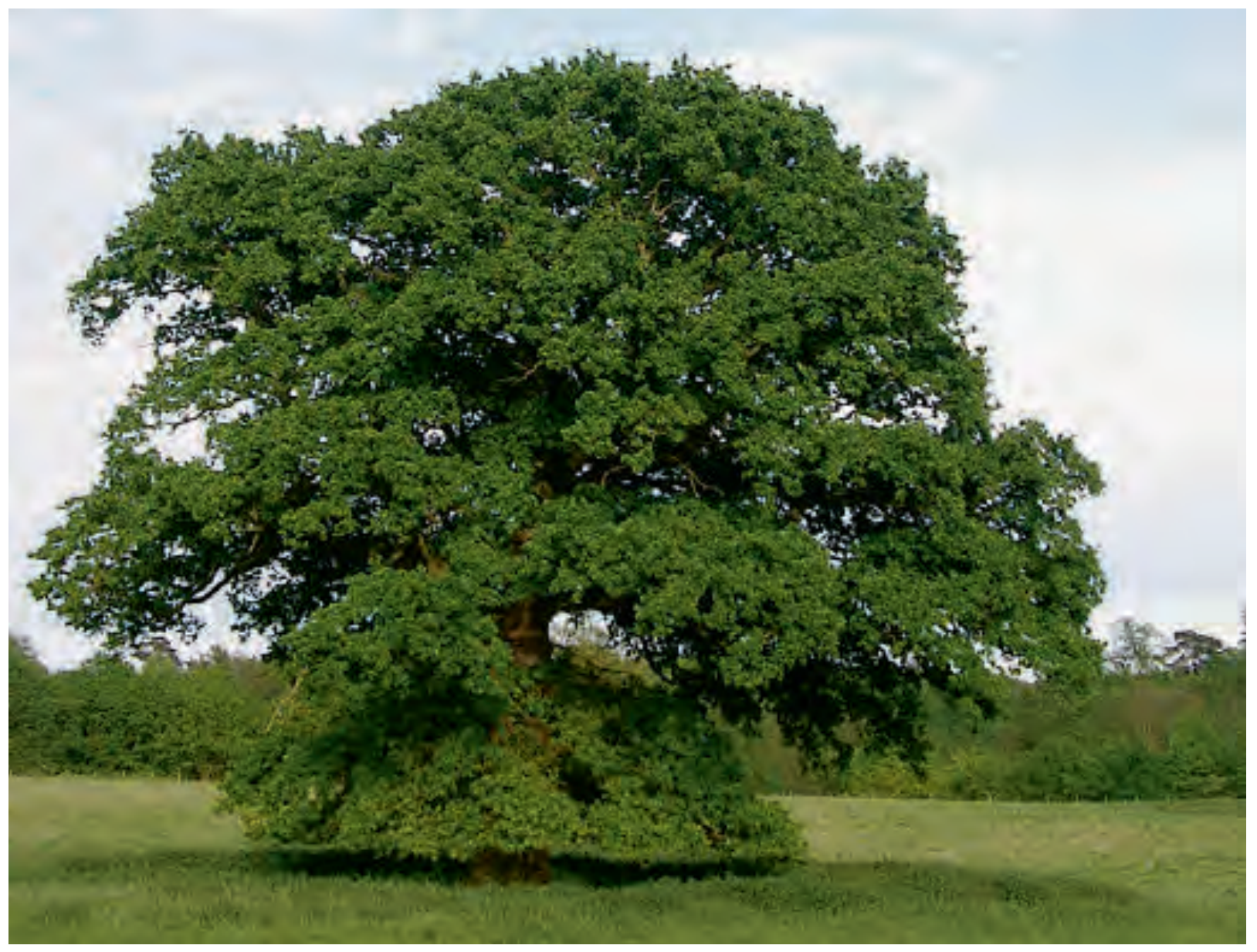




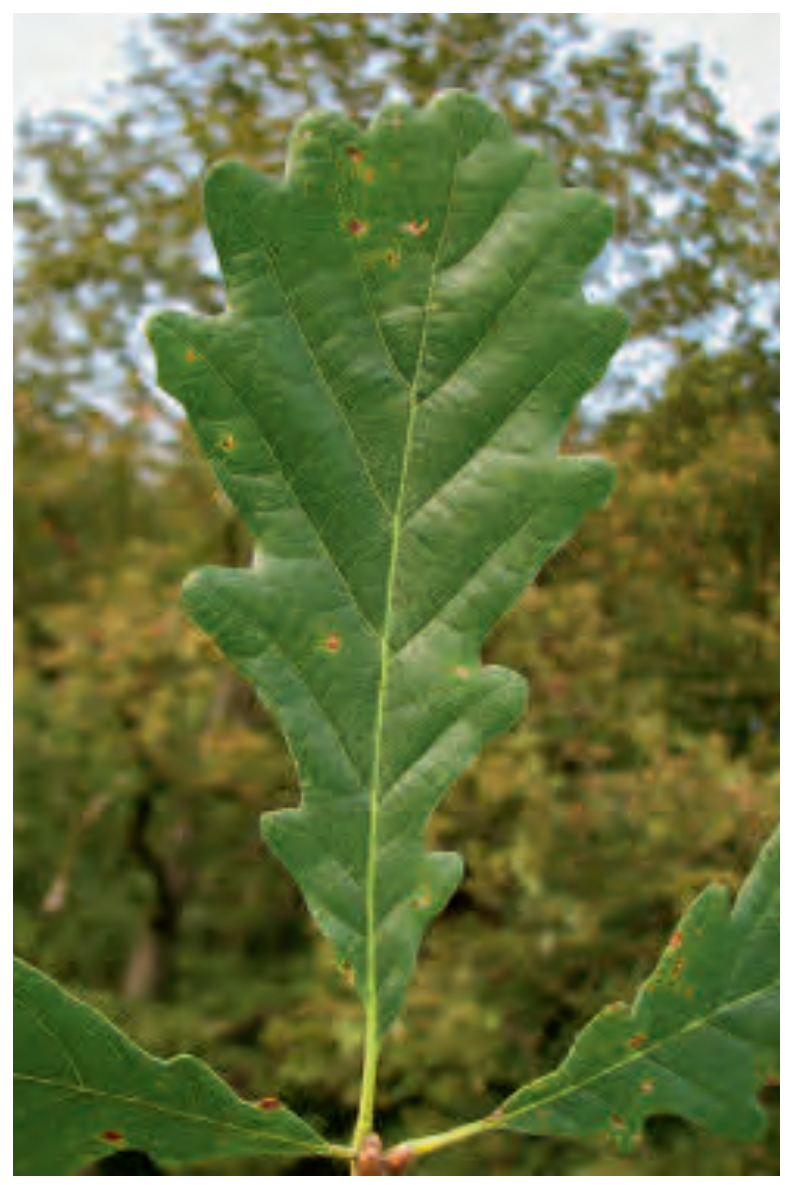

gern bevorzugt gefressen, sondern auch von vielen Vogelarten (z. B. Eichelhäher). Das Totholz ist ein wichtiges Habitat für den Hirschkäfer. Eichen stellen somit wichtige Gehölze im Ökosystem Wald dar.

\section{Systematik und Verbreitung}

Die Trauben-Eiche gehört zu den Buchengewächsen (Fagaceae) und hier zur Unterfamilie der Quercoideae, den Eichenartigen. Hierzu werden außerdem die Gattungen Castanea, Castanopsis, Chrysolepis, Lithocarpus und Trigonobalanus gestellt, von denen nur die erste mit der Esskastanie (Castanea sativa) in Deutschland heimisch ist (Mabberley 2008). Quercus umfasst rund 450 Arten, die überwiegend in gemäßigten bis subtropischen Bereichen Eurasiens, in N-Afrika und N-Amerika bis ins tropische westliche S-Amerika verbreitet sind (Roloff \& Bärtels 1996). Neben der bereits genannten Stiel-Eiche tritt in Deutschland im sehr milden Weinbauklima des Südwestens Deutschlands noch die Flaum-Eiche (Quercus pubescens) auf. Die Trauben-Eiche ist in weiten Teilen Europas bis Kleinasien verbreitet und fehlt nur im Nordosten und Osten. Das Areal ist jedoch kleiner als das der Stiel-Eiche und liegt mehr im atlantisch geprägten, milderen Klima (Sснütт et al. 2002). Die Trauben-Eiche ist ein typisches Element in Eichenmischwäldern, Feldgehölzen und Hecken (Kiermeier 1993). Sie kommt dabei vom Tiefland bis in die Mittelgebirge vor. Ursprünglich fehlte sie an der Nordseeküste sowie auf den Nordseeinseln, in Teilen des nördlichen Alpenvorlandes und in den nördlichen Kalkalpen. Sie wächst dort auf sauren bis schwach alkalischen Substraten. Die Trauben-Eiche meidet im Unterschied zur Stiel-Eiche vernässte Standorte. Mit einer Verträglichkeit gegenüber sandig-lehmigen, schottrigen, steinigen und flachgründigen Substraten bis hin zu reinem Lehm ist sie recht bodenvag (Kiermeier 1993). Die Nährstoffansprüche der Trauben-Eiche sind deutlich geringer als die der Stiel-Eiche (Sснüтt et al. 2002). Die Schattentoleranz der Trauben-Eiche nimmt mit zunehmendem Alter rasch ab, sodass die Lichtbaumart mit deutlichem Vitalitätsverlust auf Schattendruck reagiert. Im Verbreitungsgebiet von Trauben- und Stiel-Eiche kommen zahlreiche Übergangsformen zwischen beiden Eichenarten vor, die häufig auf Bastardierungen zurückzuführen sind und dort die morphologisch variable Hybride Quercus × rosacea bilden.

\section{Habitus}

Die Trauben-Eiche erreicht nicht ganz die Größe der Stiel-Eiche, gehört aber mit Höhen bis $45 \mathrm{~m}$ dennoch zu den größten heimischen Laubbäumen. Die dickste Trauben-Eiche Europas steht in England und hat einen Stammumfang von ca. $14 \mathrm{~m}$ (Roloff 2014). Sowohl Trauben- als auch Stiel-Eiche sind ausgesprochene Pfahlwurzler. Ihr Stamm ist mehr oder weniger durchgehend und verzweigt sich nicht wie bei der Stiel-Eiche in mehrere gleichrangige Haupttriebe. Die hochgewölbte Krone ist daher

Abb. 1 (Seite 11): Alte Quercus petraea.

Abb. 2 (oben): Laubblatt. 
recht gleichmäßig aufgebaut, der Anteil an Wasserreisern ist gering. Habituell unterscheidet sich die Trauben-Eiche von der Stiel-Eiche auch dadurch, dass die Krone der Stiel-Eiche unregelmäßig gewölbt ist mit stark gedrehten unteren Ästen und einem hohen Anteil an Wasserreisern. Die Belaubung und Verzweigung im Kroneninneren ist bei der Stiel-Eiche dichter (Mitchel 1977).

Die Borke der Trauben-Eiche ist senkrecht längsgefurcht. Junge Triebe sind dunkelgrau, gräulich bereift und sonnenseits oft gerötet. Die Winterknospen sind groß, spitzkegelförmig und kantig und stehen im Spitzenbereich der Sprossachse dichter gedrängt. Sie weisen zahlreiche feine weiß behaarte Knospenschuppen auf, deren Spitze meist dunkel- bis schwarzbraun gefärbt ist.

In trockenen Jahren ist besonders im Sommer und Spätsommer ein Abwurf beblätterter, kleinerer Äste zu beobachten. Dieser Abwurf ist nicht krankheitsbedingt, sondern repräsentiert einen Schutzmechanismus der Pflanze, um die transpirierende Oberfläche einzuschränken. Dabei erfolgt der Abwurf an einer Art Sollbruchstelle im Übergangsbereich zwischen der Seitentriebbasis und der Abstammungsachse, wie es auch auch bei Vogel-Kirschen, Pappeln und Weiden zu beobachten ist.

\section{Belaubung}

Quercus petraea ist eine winterkahle Art, die ihre derb-ledrigen Blätter nach einer gelbbraunen bis braunen Herbstfärbung nicht unmittelbar abwirft. Besonders an jüngeren Pflanzen bleiben die eingetrockneten Blätter bis zum kommenden Frühjahr erhalten und werden erst zum Zeitpunkt des Neuaustriebs abgeworfen. Die Blätter haben einen 1-2 cm langen Blattstiel und einen keilförmigen Spreitengrund, im Unterschied zu Quercus robur mit kurzem Blattstiel und geöhrtem Spreitengrund. Die zwei fadenartigen Nebenblätter sind kurzlebig und werden oft nur rudimentär ausgebildet.

Abb. 3 (oben): Zweig mit Knospen.

Abb. 4 (unten): Unreife, noch grüne Eicheln.
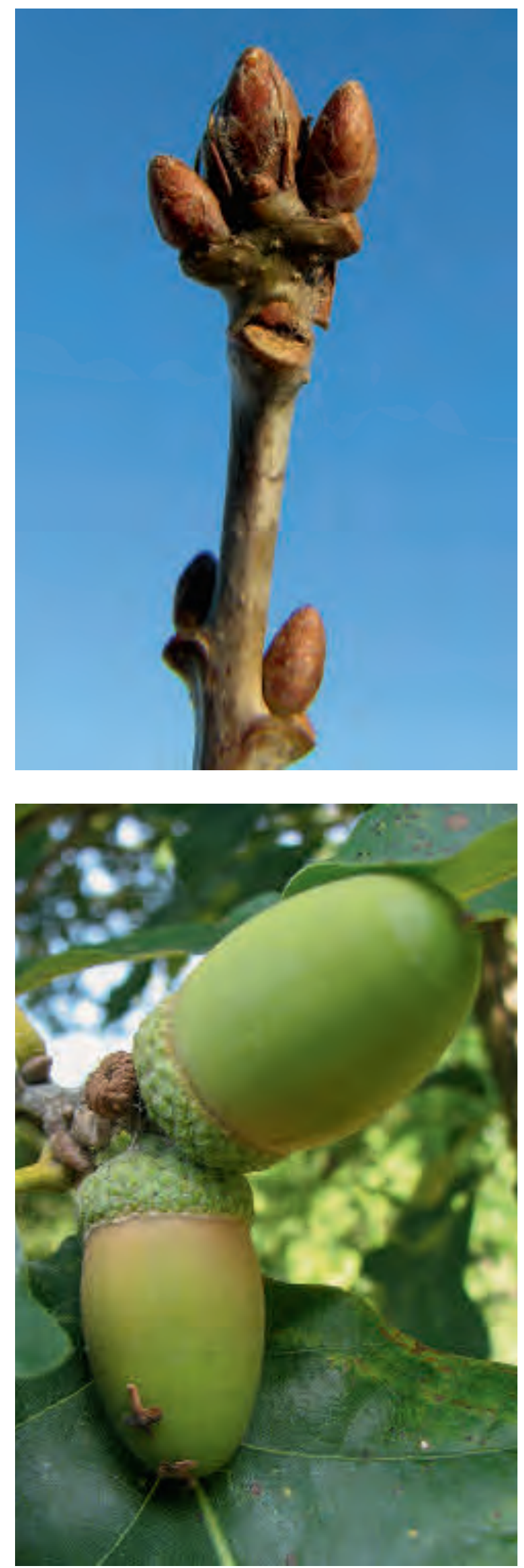

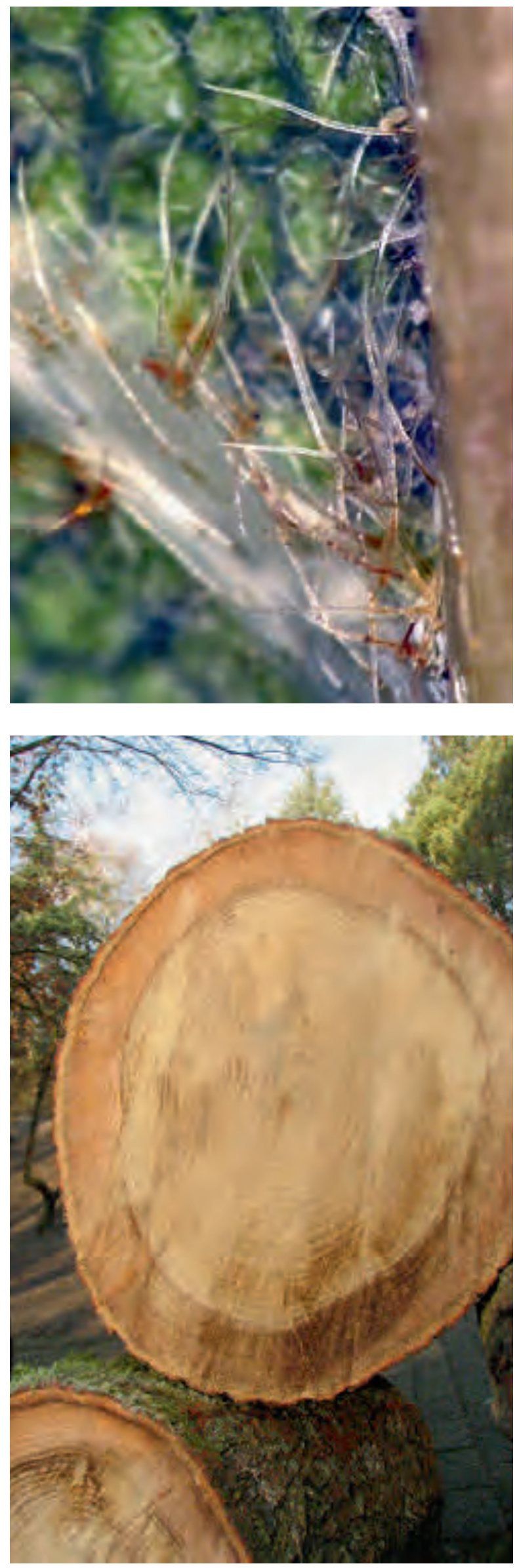

Die Blattspreite der verkehrt eiförmigen Blätter weist 4-9 Paare regelmäßige und runde Lappen auf. Der Blattstiel ist wie auch die Mittelrippe gelblich. Die Blätter stehen mehr oder weniger regelmäßig an der Sprossachse verteilt (Unterschied zu Quercus robur: dort am Triebende dicht büschelig). Nervenwinkel wie auch die Bereiche entlang der Mittelrippe sind mit Büschelhaaren besetzt, im Unterschied zu Quercus robur, bei der sie kahl sind. Bei der Trauben-Eiche fehlen Buchtennerven, während diese bei der Stiel-Eiche besonders deutlich entwickelt sind (Roloff 2014). Der Blattaustrieb erfolgt bei der Tauben-Eiche rund zwei Wochen vor dem Austrieb der Stiel-Eiche.

\section{Blüten}

Wie alle übrigen Eichen-Arten ist die TraubenEiche windblütig. Daher ist ihre Blütenhülle stark reduziert und die Blüten sind unscheinbar. Die Art ist einhäusig, männliche und weibliche Blüten stehen in getrennten Ständen auf der Pflanze verteilt. Die Blüten erscheinen mit dem Laubaustrieb Ende April bis Anfang Mai. Der Blütezeitpunkt der Trauben-Eiche liegt ungefähr zwei Wochen vor dem der Stiel-Eiche (Kiermeier 1993). In den weiblichen Blütenständen stehen 2-3 kurz gestielte bis sitzende Blüten dicht gedrängt traubig beieinander, daher die deutsche Bezeichnung Trauben-Eiche. Bei der Stiel-Eiche stehen die Blüten und später auch die Früchte auf bis zu $12 \mathrm{~cm}$ langen Stielen. Bei den weiblichen Blüten sind die drei Narben leuchtend rot gefärbt. Der Fruchtknoten ist 3-fächerig. Im Unterschied zu den weiblichen Blütenständen, die eher im terminalen Bereich der Triebe ausgebildet werden, stehen die männlichen mehr am Grund von Langtrieben. Die männlichen Blüten sind in 7-12 cm langen hängenden Kätzchen angeordnet.

Abb. 5 (oben): Ausschnitt aus der Blattunterseite mit Haaren in den Winkeln der Adern.

Abb. 6 (unten): Eichenstamm mit deutlichen Jahresringen.

Abb. 7 (Seite 15): Gruppe älterer Eichen im Sauerland mit gerade frisch austreibendem Laub. 


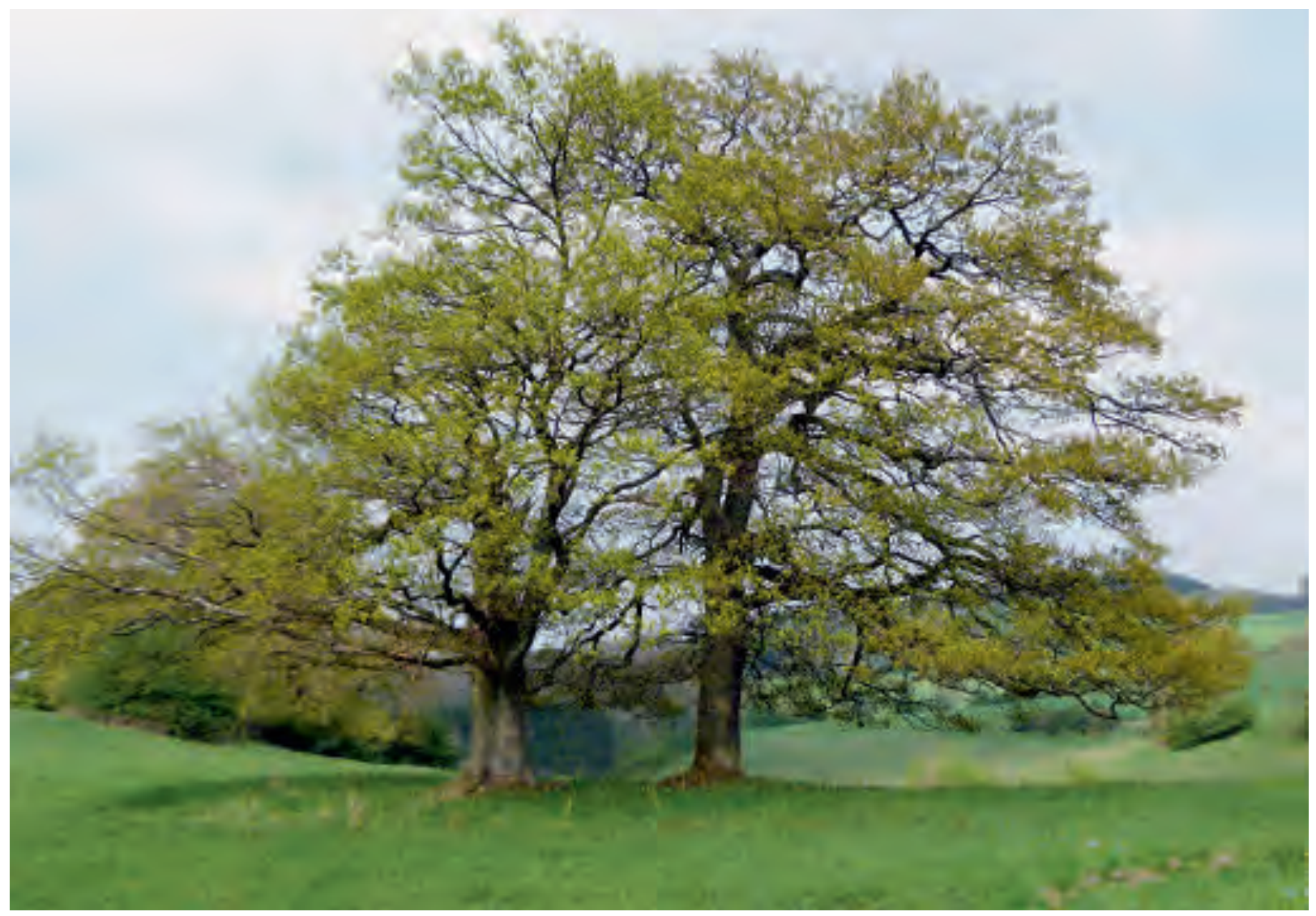

\section{Früchte}

Bei den Eicheln handelt es sich um einsamige Nussfrüchte, die von einer Hülle, der sog. Cupula, umgeben sind. Diese Hülle wird als Verzweigungssystem gedeutet, bei dem die beteiligten Sprossachsen zu einer becherartigen Struktur verschmolzen sind. Die deutlich erkennbaren Schuppen an der Außenseite der Cupula sind Tragblattrudimente nicht fruchtender Zweige. Bei Eichen ist die Cupula becherförmig, bei Buchen (Fagus) öffnet sie sich vierklappig. Im Unterschied zu fremdländischen Eichenarten erfolgt bei der TraubenEiche die Fruchtreife im Jahr der Blüte. Die Eicheln der Trauben-Eiche sind 1,3-2,5 cm lang, ca. $1 \mathrm{~cm}$ breit und nur kurz gestielt. Bei Quercus robur wird die Fruchtstandsachse bis $12 \mathrm{~cm}$ lang. Die Fruchtreife erfolgt von Ende September bis Oktober. Auf den Früchten sind im Unterschied zur Stiel-Eiche keine dunklen Längsstreifen zu erkennen. Sie sind bei der Reife etwa ein Viertel von der Cupula umgeben, bei der Stiel-Eiche sind es ein Drittel oder die Hälfte. Trauben-Eichen bilden im Vergleich $\mathrm{zu}$ anderen heimischen Baumarten erst in einem späten Alter die ersten Eicheln, im dichten Bestand erst nach ca. 60 Jahren (HöLl 1983). Die Früchte werden besonders von Eichelhäher und Eichhörnchen gesammelt und für den Winter versteckt. $\mathrm{Zu}$ Boden gefallene Eicheln rollen nach dem Aufprall noch einige Meter weiter. Nach einem sehr produktiven Jahr folgt meist eine Pause von 1-2 Jahren, bevor wieder massenhaft Früchte produziert werden. In bestimmten Regionen wurden Eicheln früher und werden auch heute noch als Schweinefutter verwendet. Schweine wurden zum Fressen der Eicheln in die Wälder getrieben. Daher bezeichnet man die Jahre, in denen massenhaft Früchte gebildet werden, auch als Mastjahre.

\section{Krankheiten und Schädlinge}

Auch wenn die Trauben-Eiche vergleichsweise widerstandsfähig gegenüber Krankheiten ist, wird sie von verschiedenen pathogenen Insekten und Pilzen befallen. Gegenüber dem Eichenwickler ist die Art relativ resistent (Miтchel 1977), durch starken Befall von Schwammspin- 


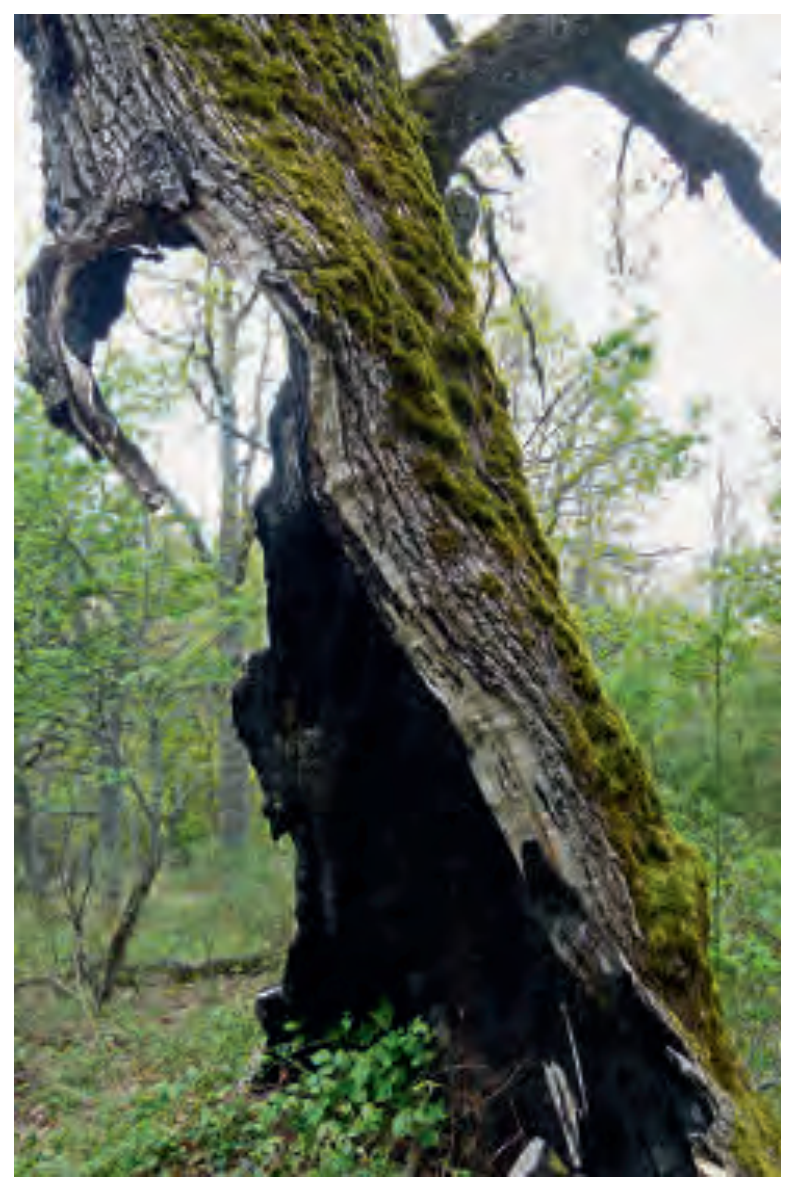

ner und besonders durch den Frostspanner, dem Hauptfressfeind der Trauben-Eiche, kann es allerdings zum vollständigen Kahlfraß kommen. Dieser wird von der Pflanze durch einen starken zweiten Austrieb in Form der Ausbildung von Johannistrieben kompensiert. Zum Zeitpunkt des Austriebes ist im Eichenlaub der Gehalt an Tanninen als Fraßschutz besonders hoch; mit zunehmendem Alter des Blattes nimmt er ab. Die an den Blättern der Eichen häufig zu beobachtenden runden Galläpfel der Eichengallwespe (Cynips quercusfolii) stellen für das Blatt selbst kein Problem dar und das Blatt verliert durch diesen Befall nicht seine Funktion. In den letzten Jahren ist ein weiterer Schädling, der Eichenprozessionsspinner, an Eichen zu beobachten, der nicht nur für die Eichen selbst ein Problem darstellt, sondern auch für den Menschen. Die Gifthaare des Eichenprozessionsspinners können starke allergische Reaktionen vor allem der Haut hervorrufen. In besonders feuchten Jahren wird das junge Laub durch Mehltau befallen, ein Pilz,

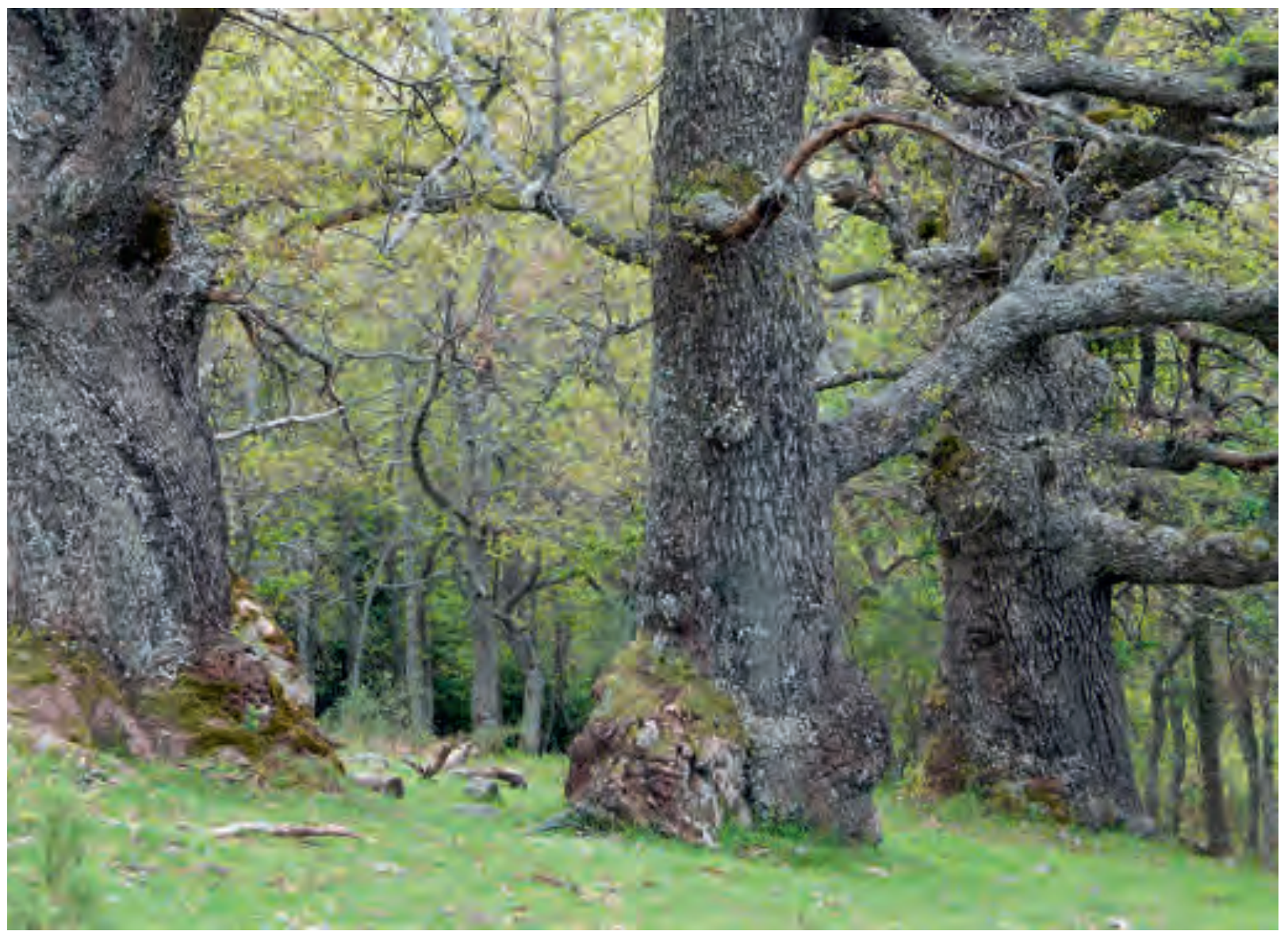


der sich in Form eines weißen mehlartigen Überzugs darstellt.

\section{Verwendung}

Die Trauben-Eiche ist eine vielseitig einsetzbare Baumart. Neben der Verwendung als Dorfund Hofbaum eignet sie sich hervorragend als Straßen- und Alleebaum sowie als Solitärbaum in Parkanlagen. Da sich die Trauben-Eiche nach einem auf den Stock setzen durch zahlreiche Stockausschläge wieder regeneriert, ist sie auch hervorragend zur Anlage von Baumhecken geeignet. Die Trauben-Eiche ist ein weit verbreiteter Forstbaum und Lieferant von vielseitig nutzbarem Holz und stellt zusammen mit der Rot-Buche und der Stiel-Eiche die wichtigste heimische, forstlich genutzte Laubbaumart dar. Das Holz von Trauben- und Stiel-Eiche ist kaum voneinander zu unterscheiden und wird daher auch entsprechend gleich genutzt. Das Holz weist einen dunkleren Kernholz- und einen helleren weißlichen Splintholzbereich auf. In dem ringporigen Holz sind deutliche Jahresringe zu erkennen. Das Holz lässt sich in mild und hart einteilen. Mildes Eichenholz ist engringig und hat einen hohen Anteil an Frühholz. Es wird zur Herstellung von hochwertigen Furnieren und Massivholzmöbeln genutzt, findet aber auch Verwendung im Innenausbau sowie für Drechsel- und Schreinerarbeiten (ScHüTT et al. 2002). Hartes Eichenholz ist breitringig und weist einen hohen Anteil an Spätholz auf. Es wird besonders als Bau- und Konstruktionsholz geschätzt, aber auch im Treppen- und Wasserbau sowie zur Herstellung von Bahn-

Abb. 8 (Seite 16 oben): Selbst wenn alte Eichen durch Blitze getroffen werden und ein Großteil des Stammes verbrannt ist, können sie noch weiterleben (Picos de Europa).

Abb. 9 (Seite 16 unten): In atlantischen Gebieten mit mild-feuchtem Klima gedeihen Eichen sehr gut. Mehrere hundert Jahre alte Trauben-Eichen im Nationalpark Picos de Europa in Nordspanien.

Abb. 10 (oben): Durch Verletzungen entstehen bei alten Eichen mitunter sehr bizarre Wuchsformen (Picos de Europa).

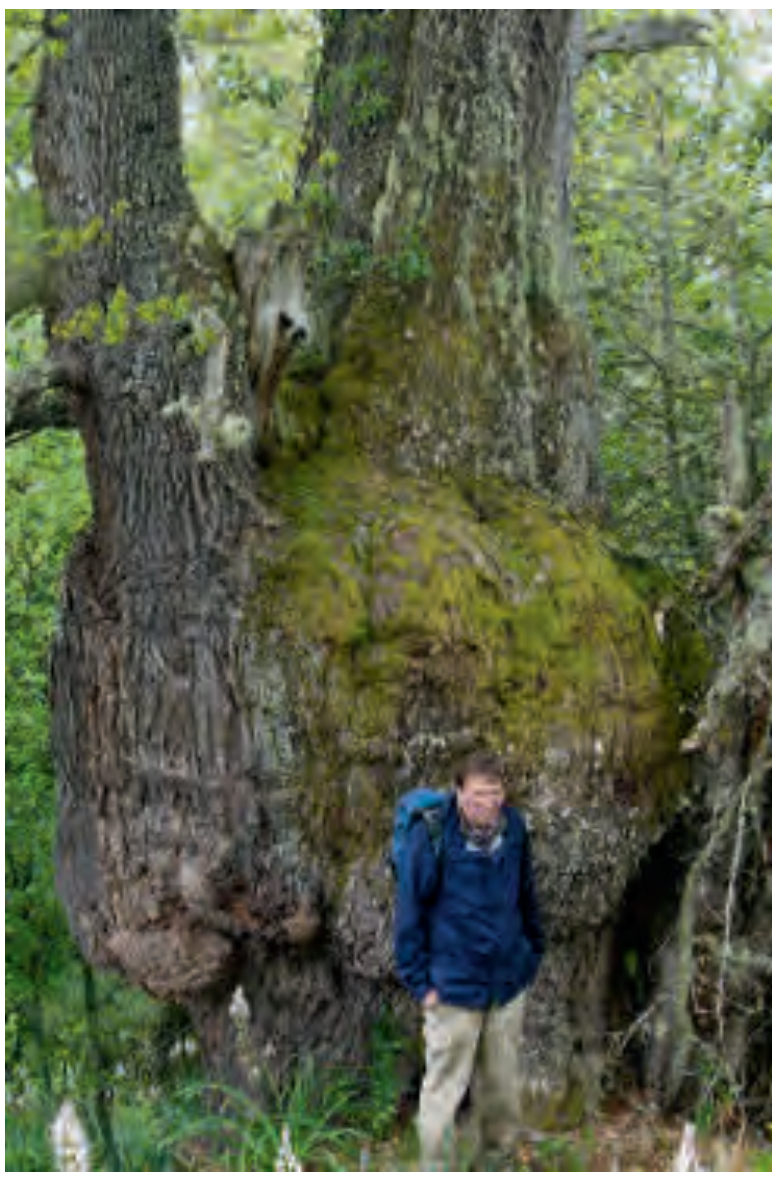

schwellen und hochwertigen Parketten. Aus Eichenholz lassen sich außerdem robuste Fässer herstellen, in denen heranreifende Whiskeyund Weinsorten ihre spezielle Geschmacksnote erhalten. Die aus der Borke gewonnenen Tannine werden besonders in der Ledergerberei und Homöopathie eingesetzt.

\section{Literatur}

Düll, R. \& Kutzelnigg, H. 2011: Taschenlexikon der Pflanzen Deutschlands und angrenzender Länder, 7. Aufl. - Wiebelsheim.

Höll, W. 1983: Bäume in Mitteleuropa. - Köln. Kiermeier, P. 1993: Wildgehölze des mitteleuropäischen Raumes, BdB-Handbuch, Teil VIII, 5. Aufl. - Pinneberg. Mabberley, D. J. 2008: Mabberley's plant book, ed. 3. Cambridge.

Mitchel, A. 1979: Die Wald- und Parkbäume Europas. - Berlin \& Hamburg.

Roloff, A. 2014: Baum des Jahres 2014, die TraubenEiche (Quercus petraea (Matt.) Lieb.). - Ginkgoblätter 134: 25-29.

Roloff, A. \& Bärtels, A. 1996: Gartenflora Bd. 1.

- Stuttgart.

Sснüтt, P., Sснuck, H. J. \& Sтimm, B. 2002: Lexikon der Baum- und Straucharten. - Hamburg. 